\section{Nonmetric analysis of difference relations in triangular designs}

\author{
STANLEY J. RULE \\ University of Alberta \\ Edmonton, Alberta, Canada
}

The present paper illustrates an application of a general procedure for performing nonmetric analyses. The method can be used to estimate parameters of nonlinear functions of real-valued independent variables as well as to solve for scale values of scaling models. The algorithm is called NOPE (Rule, 1979), an acronym for NOnmetric Parameter Estimation. It offers the advantage that the user can specify the equation to be fitted to the rank order of the data; consequently, he or she is not limited to fitting models that have been preselected by the programmer.

In the present methodological study, values of a difference model were recovered from data arranged in a triangular design. Triangular designs are often employed to analyze judgments of differences for only one permutation of the two stimuli in each pair. That is, two stimuli $A$ and $B$ may appear in the order $A B$ but not in the order $B A$. As a consequence, pairs of $n$ stimuli yield $n(n-1) / 2$ data in a triangular design, as compared with $n^{2}$ data in a complete square design. The missing data in a triangular design introduce the problem for nonmetric analysis of how to fit models that are not symmetrical with respect to the positions of the stimuli in a pair-that is, models for which values assigned to a stimulus depend upon whether the stimulus appears first or second.

In a typical application of a triangular design, the subject is asked to judge the positive difference for each combination of two stimuli from a set of stimuli. At times, the experimental question does not permit the collecting of negative differences. For example, in a test of the twostage model of magnitude estimation, Rule, Curtis, and Markley (1970) obtained judgments of differences under magnitude-estimation instructions. Because negative differences are not defined for magnitude estimation, only positive differences were evaluated in a triangular arrangement. A similar example is provided by Popper, Parker, and Galanter (1986), who asked subjects to match the loudness of a single tone to the difference in loudness of a pair of tones. Because it is not possible to set a negative loudness, only positive settings were analyzed in a triangular arrangement.

Usually when difference judgments in a triangular design are subjected to a nonmetric scaling analysis, the model fitted to the ordinal data is of the form

This research was supported by Grant A0151 from the Natural Sciences and Engineering Research Council of Canada. Requests for reprints should be sent to Stanley J. Rule, Department of Psychology, University of Alberta, Edmonton, Alberta T6G 2E9, Canada.

$$
Z_{i j}=V_{j}-V_{i}
$$

In Equation $1, V_{i}$ and $V_{j}$ are scale values associated with the $i$ th and $j$ th stimuli, and $Z_{i j}$ denotes values of the model that are as close as possible to a monotonic transformation of the difference judgments. The symmetric model presented as Equation 1, in which the first and second stimulus in each pair are given equal weight, presents no difficulty to a nonmetric analysis of triangular data. However, as Birnbaum (1981) has noted, experimental effects such as time-order error may enter into the relation, and some asymmetric expression rather than Equation 1 may provide the appropriate model for data analysis. Birnbaum fitted a symmetric model (the absolute value of the expression given as Equation 1) to triangular data that had been generated by an asymmetric model in which the first and second stimuli were weighted unequally. As is to be expected, the fitting of the incorrect model to the ordinal data failed to recover the parameters that generated the data.

The question addressed in the present paper is how to analyze ordinal data in a triangular design when the appropriate scaling model is an asymmetric model of the form

$$
Z_{i j}=V_{j}-b V_{i},
$$

in which the coefficient $b$ is not equal to 1.0. An unequal weighting of stimuli has been fitted by a metric method to triangular data from judgments of differences in duration, to account for the time-order error often found in duration judgments (Curtis \& Rule, 1977). A difficulty arises in adapting nonmetric procedures to solve such expressions as Equation 2, because the data in a triangular design implicate fewer ordinal constraints than those in a complete square design.

In the present paper, three approaches to the problem are compared. The methods are illustrated by solutions to artifical data, from which the estimates of parameters from a triangular design are compared with those of a square design containing the same number of data. The data were generated from the relation

$$
Y_{i j}=X_{j}^{k}-b X_{i}^{k},
$$

where $X$ denotes hypothetical measures of physical stimuli. Estimates of the exponent $k$ and coefficient $b$ from the ordinal data were compared with their parametric values that generated the data.

The three analytic procedures compared in the study differed in the routes taken to solve Equation 3. Two approaches consisted of nonmetric scaling analyses in a first step, followed by the fitting of a power function to the relation between scale values and $X$ by full metric procedures in a second step. The remaining, totally nonmetric, approach bypassed the scaling step and recovered the parameters of the psychophysical power function directly 
from the ordinal data. By estimating parameters directly from an order, a totally nonmetric approach avoids the cumulation of error entailed in sequential analyses and, consequently, would be expected to provide the most stable estimates of parameters. Alternatively, analytic procedures that yield scale values as an intermediate step to estimating parameters have the advantage of permitting the evaluation of predicted trends at each step in the analysis.

\section{Artificial Data}

Artificial data were generated with Equation 3 by setting the exponent $k$ equal to 0.8 and the coefficient $b$ equal to 1.12. Although these parameter values are arbitrary, they were selected to be in the neighborhood of what one might expect to find in data from psychophysical research. For the square design, a set of eight values of $X$ was crossed with itself to yield 64 data. The values of $X$ ranged from 0.5 to 10.0 , in equal steps of 1.36 . For the triangular design, 12 values of $X$ were selected, which ranged from 0.5 to 10.0 in equal steps of 0.86 . Two entries were omitted from the triangular design-the pairing of the lowest value of $X$ with the second lowest value and the pairing of the greatest value of $X$ with the second greatest value - which resulted in 64 entries, the same number of data as was generated for the square design. Figure 1 presents plots of the data obtained by substituting the values of the parameters for $b$ and $k$ in Equation 3 for the square and triangular designs.

The order of errorless data such as those plotted in Figure 1 can also be generated from a narrow range of values of the function's parameters. For the present examples, the order of the triangular data is invariant for values of $k$ ranging from 0.797 to 0.804 and values of $b$ ranging from 1.119 to 1.122 , and the order of the square data is invariant for corresponding values of $k$ and $b$ ranging from 0.789 to 0.808 and from 1.112 to 1.126 . The smaller range for the triangular data is due to a closer spacing of the independent variable $X$, which was used in order to generate the same number of data for the two designs. Had the data analyzed for the triangular design corresponded to the triangular part of the data in the square design, a greater range of values would generate the same order. Also, because of the reduced number of data, a greater range of estimates of parameters would be expected than what is reported below for the triangular design. A minimum-stress solution to fallible data, in contrast with errorless data, yields unique estimates of the parameters in Equation 3.

The data to be analyzed were generated in the same manner as in Rule (1979). A random Gaussian deviate was added to each computed value of the function given in Equation 3. The deviate was selected from a population with a standard deviation of $10 \%$ of the standard deviation of the unperturbed values. A standard error of estimate equal to $10 \%$ of the standard deviation of the unperturbed values corresponds to an $r^{2}$ of .99 between unperturbed values and the fallible data. This level of error was selected to be similar to what has been observed in real experiments of this type, and it allowed comparisons with previous analyses (Rule, 1979) of a variety of expressions in which this level of error was used. One hundred such sets of data were generated for each design.

\section{Results}

Solution 1. The first procedure followed an indirect route to solve for estimates of parameters $k$ and $b$ in Equation 3. The data were first subjected to a nonmetric scaling analysis to obtain separate sets of scale values for the first and second stimuli in each pair. The coefficient $b$ was estimated from the relation between the two sets of scale values, and the exponent $k$ was estimated from the relation between scale values and the hypothetical stimulus values of $X$.
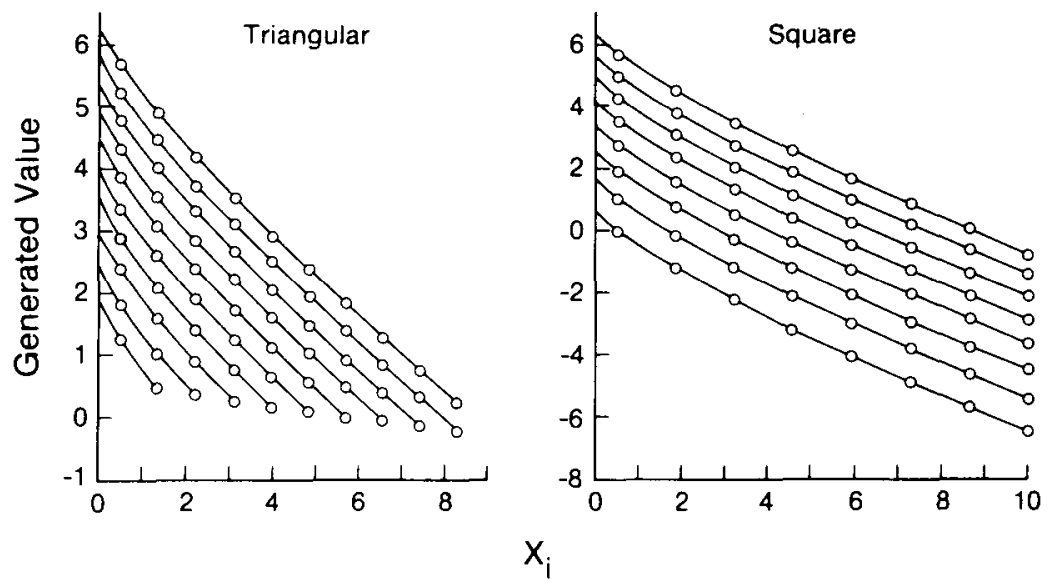

Figure 1. Artificial data for triangular and square designs generated from Equation 3 for $k=0.8$ and $b=1.12$. The data are plotted as a function of values of $X$ appearing as the second argument in the equation. Lines connect points associated with common values of $X$ appearing as the first argument. 

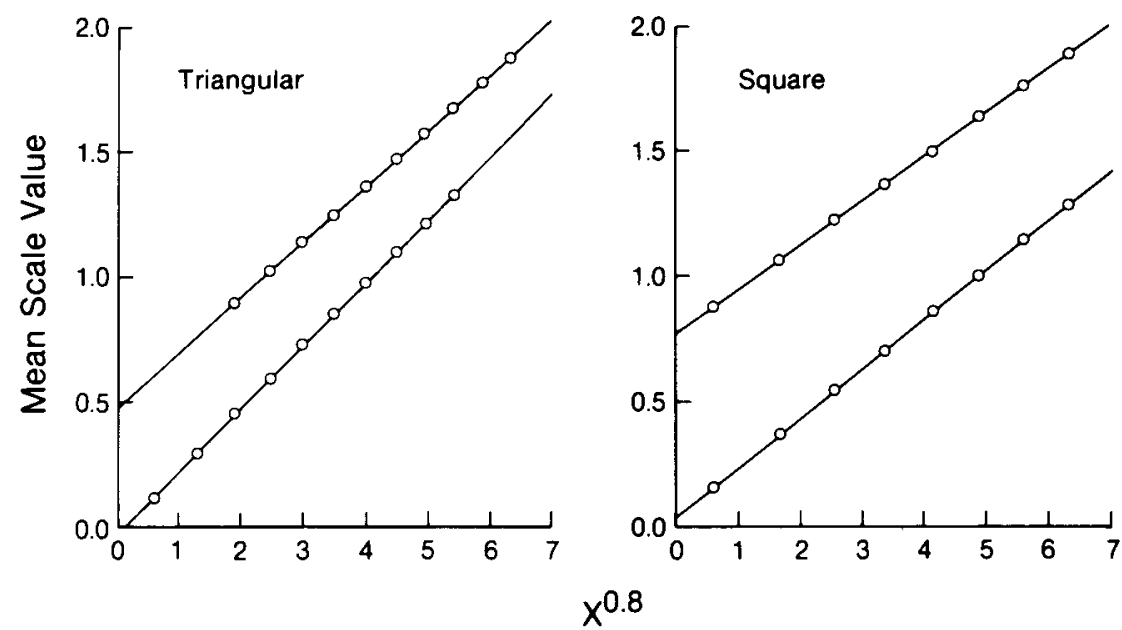

Figure 2. Solution 1: Mean scale values for the first and second stimuli derived from data in triangular and square designs, plotted as a function of values of the independent variable raised to the 0.8 power.

The scaling model fitted to the ordinal data can be expressed as

$$
Z_{i j}=V_{j}-W_{i}
$$

In the analysis of data in the triangular design, the scale values denoted by $V$ were always associated with the greater stimulus in a pair, and the scale values in Equation 4 denoted by $W$ were always associated with the lesser stimulus. It is not possible to estimate all 12 values of $V$ and $W$, because of the missing data in the triangular design. Values of $V$ cannot be estimated for the lowest two stimuli, and values of $W$ cannot be estimated for the highest two stimuli. Hence, a nonmetric solution yields 10 values each for $V$ and $W$, of which 8 are associated with the same stimuli. With respect to the square design, values of $V$ and $W$ were estimated for the eight hypothetical stimuli that generated the data.

Solutions for scale values $V$ and $W$ were obtained from Roskam's (1977) UNICON, a computer program for solving for scale values for simple polynomial models. It uses a numerical method similar to Kruskal's (1965) MONANOVA. The measure of stress, $S$, that was minimized by the solution is expressed as

$$
S=\sqrt{\frac{\sum\left(Z_{i j}-\bar{Z}_{i j}\right)^{2}}{\Sigma\left(Z_{i j}-\bar{Z}\right)^{2}},}
$$

where $\hat{Z}_{i j}$ denotes fitted values obtained as part of the analysis that are a monotonic transformation of the data and a least-squares approximation to values of the function $Z_{i j}$. The average scale values from the 100 sets of artificial data are plotted in Figure 2 against the values of the independent variable raised to the 0.8 power. In each case, the average scale values were recovered to within a linear transformation.

The linearity of the curves in Figure 2 allows the recovery of the coefficient $b$ in Equations 2 and 3. Because scales $V$ and $W$ share a common unit, an estimate of the coefficient $b$ can be obtained from the slope of $W$ on $V$ for scale values associated with the same stimuli. For the present case, estimates of $b$ were computed as the ratio of the standard deviations for the eight values of $W$ and $V$ for which the stimuli were in common. This procedure does not depend on any assumed relation between scales and measures of the independent variable. Hence, it is applicable for cases in which no relation has been specified or in which applicable physical measures of stimuli are not available. The mean estimates of $b$ computed in this fashion for each of the 100 samples and standard errors of the estimates of $b$ computed as deviations from its parametric value (i.e., from 1.12) are presented in Table 1 , together with estimates from other analyses. For each design, the mean estimate is close to its parametric value, with the analysis of the square design providing the smaller standard error.

Table 1

Mean Estimates and Standard Errors for 100 Parameter Estimates from Three Solutions, and Mean Stress for Each

\begin{tabular}{|c|c|c|c|c|}
\hline \multirow[b]{2}{*}{ Parameter } & \multicolumn{2}{|c|}{ Triangular } & \multicolumn{2}{|c|}{ Square } \\
\hline & $M$ & $S E$ & $M$ & $S E$ \\
\hline \multicolumn{5}{|c|}{ Solution 1} \\
\hline $\begin{array}{l}k_{1} \\
k_{2} \\
b\end{array}$ & $\begin{array}{r}.811 \\
.790 \\
1.117\end{array}$ & $\begin{array}{l}.103 \\
.049 \\
.050\end{array}$ & $\begin{array}{r}.807 \\
.797 \\
1.117\end{array}$ & $\begin{array}{l}.070 \\
.059 \\
.037\end{array}$ \\
\hline Stress & .041 & .007 & .044 & .008 \\
\hline \multicolumn{5}{|c|}{ Solution 2} \\
\hline $\begin{array}{l}k \\
b\end{array}$ & $\begin{array}{r}.797 \\
1.118\end{array}$ & $\begin{array}{l}.045 \\
.047\end{array}$ & $\begin{array}{r}.800 \\
1.117\end{array}$ & $\begin{array}{l}.039 \\
.036\end{array}$ \\
\hline Stress & .049 & .007 & .051 & .007 \\
\hline \multicolumn{5}{|c|}{ Solution 3} \\
\hline $\begin{array}{l}k \\
b\end{array}$ & $\begin{array}{r}.787 \\
1.107\end{array}$ & $\begin{array}{l}.038 \\
.037\end{array}$ & $\begin{array}{r}.802 \\
1.119\end{array}$ & $\begin{array}{l}.039 \\
.037\end{array}$ \\
\hline Stress & .060 & .005 & .056 & .006 \\
\hline
\end{tabular}
Solution, for Data in Triangular and Square Designs 


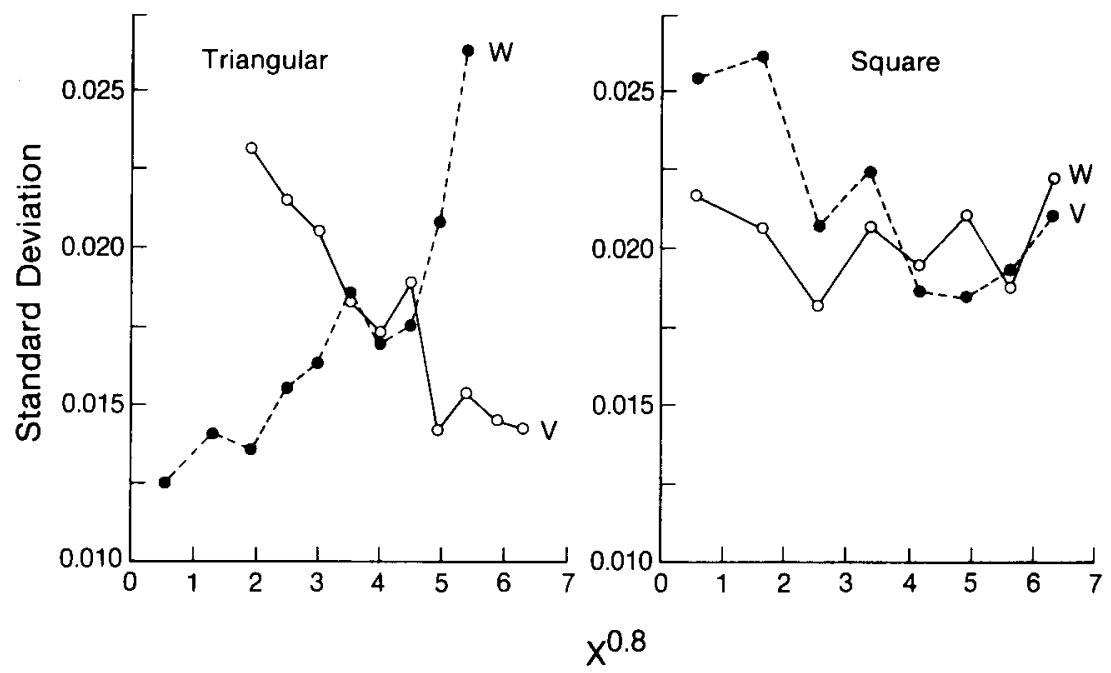

Figure 3. Solution 2: Standard deviations of scale values for the first $(V)$ and second $(W)$ stimulus, plotted as a function of values of the independent variable raised to the 0.8 power.

Two estimates of the exponent $k$ were obtained, one for each set of scale values. The estimates were computed with the Gauss-Newton method of fitting a nonlinear regression function to a least-squares criterion (Hartley, 1961). The expressions fitted to scale values were

$$
V_{i}=a_{1} X_{i}^{k}+c_{1},
$$

and

$$
W_{j}=a_{2} X_{j}^{k}+c_{2},
$$

where $c_{1}$ and $c_{2}$ allow for the arbitrary origins of the scales. Mean estimates of the exponents and their associated standard errors are presented in Table 1. Each estimate is close to the parametric value of 0.8 . The lower standard errors favor the square design, as would be expected.

Figure 3 presents a plot of the standard deviation of the 100 estimates of each scale value against its corresponding value of the independent variable raised to the 0.8 power. For the triangular design, there is an asymmetry in the relation between standard deviations and scale values. The standard deviations decrease with $V$ and increase with $W$. This asymmetry in estimates reflects differences in the frequency with which a stimulus appears as the first or second member of a pair in the triangular design. Lower standard deviations are associated with stimuli that appear with greater frequency. An asymmetry also appears in Table 1 for the triangular design with respect to the standard errors for estimates of the exponent $k$, with the estimate associated with scale values of $W$ appearing to be the more stable.

Solution 2. The second approach to solving Equation 3 also involved nonmetric scaling in an initial step. However, it differed from the first solution in that a single set of scale values of $V$ was obtained for the first and second stimuli in the pairs together with an estimate of the coefficient $b$ in the initial scaling step. Solving for a single set of scale values avoids the asymmetries in stability of estimates due to unequal frequencies with which the scale values appear in the triangular set of data. For this solution, each scale value is represented in the data with the same frequency. The scaling model fitted to the ordinal data is the expression previously presented as Equation 2:

$$
Z_{i j}=V_{j}-b V_{i}
$$

Estimates of the scale values of $V$ and the coefficient $b$ were obtained from the NOPE program (Rule, 1979) ${ }^{1}$. NOPE provides a general nonmetric procedure for solving nonlinear equations. The numerical procedure involves the monotone regression method employed in Kruskal's $(1964,1965)$ MDSCAL and MONANOVA, but replaces Kruskal's gradient method with the more general Gauss-Newton method. In previous tests of the NOPE method, Monte Carlo tests were conducted on complete data, but no solutions from triangular designs had been tested.

As in the first solution, estimates of the exponent $k$ were obtained from fitting the power function in Equation 6. Plots of the average scale values are presented in Figure 4 and estimates of the parameters are presented in Table 1, for solutions from the 100 sets of artificial data for both designs. For each design, the mean estimates are close to their parametric values, with an advantage for the square design with respect to the smaller standard errors. Note, however, that there is a tradeoff between the smaller standard errors for the square design against a greater number of scale values for the triangular design.

Solution 3. In the third approach, the scaling step was omitted, and parameters $b$ and $k$ were estimated directly from the ordinal data, using the NOPE method. The expression fitted to each set of ordinal data was

$$
Z_{i j}=a X_{j}^{k}-c X_{i}^{k} \text {. }
$$




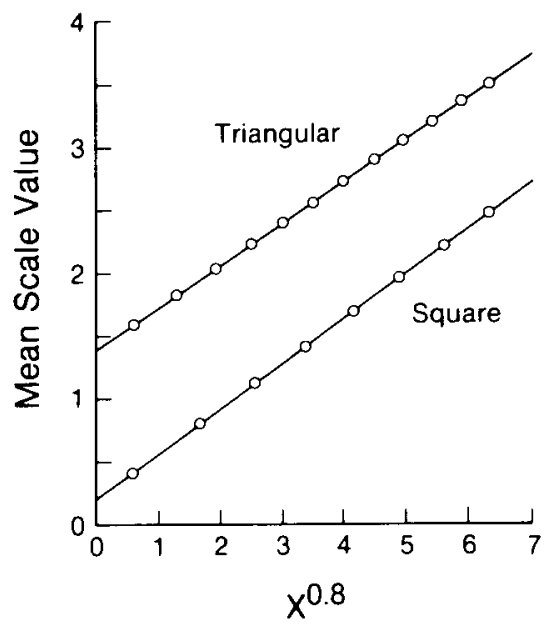

Figure 4. Solution 2: Mean scale values from fitting Equation 2 to data in triangular and square designs, plotted as a function of values of the independent variable raised to the 0.8 power.

The estimates of the coefficient $b$ were provided by computing the ratio $c / a$. Mean estimates of the parameters and their associated standard errors are included in Table 1. The results of this third solution are less sensitive to the choice of designs. The average estimates are close to the parametric values, and the standard errors are small with a slight, but not likely meaningful, advantage for the triangular design. Comparable results from fitting Equation 8 to square designs have been reported by Rule (1979), from two analyses for which the parametric values for $b$ were 0.9 and -0.8 (an additive relation) and for $k, 0.6$ and 0.4 .

The average stress of each nonmetric analysis is also presented in Table 1. As is to be expected, the stress of a solution decreases with an increase in the number of values to be estimated in the nonmetric solution. It should be noted in this regard that sometimes the stress when estimating a small number of parameters as in the third solution to Equation 8 can be much greater than what is typically considered a good fit when solving for a larger set of scale values.

\section{Discussion}

The successful recovery of parameters by each of the three solutions indicates that asymmetric models can be fitted to triangular data by nonmetric analyses. But in comparison with a square design, the pattern of results for a triangular design signals some areas of caution. Of particular concern is asymmetry in the stability of parameter estimates. This effect was evidenced in the first solution, in which separate sets of scale values were obtained for row and column variables. The opposing trends in the stability of scale values for the two scales suggest that comparisons between scales should be interpreted with care. Minor peculiarities in a set of data due only to chance variation may lead to seemingly inconsistent trends in the scales. It seems likely that the difference in the standard errors associated with estimates of the two exponents obtained in the first solution was also a consequence of the unequal weighting in deriving scales from uneven numbers of ordinal constraints. However, the problem of unequal numbers of constraints is not an issue when it is possible to use the second solution, in which a single scale was derived to measure both row and column variables.

The outcome of the example analyses also serves to illustrate interrelations among stress, stability of parameter estimates, and number of parameters. It is well known that closer fits to fallible data are obtained as the number of parameters to be estimated is increased. A relation shown in Table 1 that may not be equally well known is the tendency for standard errors of parameter estimates to increase as the number of parameters increases. It may seem paradoxical for greater stability to be associated with greater stress. But when the route taken to fit the same model to the data requires that more parameters be estimated, there is greater opportunity for estimates to be influenced by random error. This effect may be expected to be more pronounced with triangular data, because of fewer ordinal constraints within the data.

What strategy is to be followed for analyzing data depends in part on the purpose of the analysis. When the purpose is to examine the consistency of trends within the data, obtaining of scale values for each variable provides the greatest amount of information from a nonmetric procedure. In the present example, obtaining separate scales for rows and columns allowed a direct evaluation of whether or not the position of a stimulus in a pair influenced its scale value. But the price for the greater information is lower stability of the estimates of scale values for both designs. Because of the asymmetry in stability of estimates of scale values, a triangular design seems illsuited for this analysis. When the purpose of the analysis is to obtain parameter estimates to be compared with parameter values from an independent source rather than for an evaluation of internal consistency, the most stable estimates of parameters are achieved by fitting a specified function directly to the ordinal data, as in the third solution. Obviously, its use is restricted to situations in which the independent variable has an applicable physical measure, and when some function to be fitted to the data can be specified.

The second solution illustrates a strategy that provides a good compromise between the amount of information obtained from the analysis and the stability of parameter estimates. Because the solution allows for unequal weighting of row and column variables as part of the scaling analysis, it requires only one more parameter to be estimated beyond Equation 1. Furthermore, the appropriateness of a model with equal weighting can be evaluated (rather than left as an assumption) by comparing the estimate of the coefficient $b$ with unity. Clearly there is not a universally correct design or analytic strategy. The issue is one of selecting the design and method of analysis that is most appropriate to the experimental question of interest. Solutions can often be tailored to the requirements 
of the experiment by using general methods such as NOPE.

\section{REFERENCES}

Birnbaum, M. H. (1981). Reason to avoid triangular designs in nonmetric scaling. Perception \& Psychophysics, 29, 291-293.

Curtis, D. W., \& Rule, S. J. (1977). Judgment of duration relations: Simultaneous and sequential presentation. Perception \& Psychophysics, 22, 578-584

HaRTley, H. O. (1961). The modified Gauss-Newton method for the fitting of non-linear regression functions by least squares. Technometrics, 3, 269-280.

KrUSKa L, J. B. (1964). Nonmetric multidimensional scaling: A numerical method. Psychometrika, 29, 115-129.

KrUSKa L, J. B. (1965). Analysis of factorial experiments by estimating monotone transformations of the data. Joumal of the Royal Statistical Society, 27B, 251-263.

Popper, R., Parker, S., \& Galanter, E. (1986). Dual loudness scales in individual subjects. Journal of Experimental Psychology: Human Perception \& Performance, 12, 61-69.

RosKam, E. E. (1977). A survey of the Michigan-Israel-NetherlandsIntegrated series. In J. C. Lingoes (Ed.), Geometric representations of relational data (pp. 289-312). Ann Arbor, MI: Mathesis Press.

RULE, S. J. (1979). Solutions to some nonlinear equations from nonmetric data. Psychometrika, 44, 143-155.

Rule, S. J., Curtis, D. W., Markley, R. P. (1970). Input and output transformations from magnitude estimation. Joumal of Experimental Psychology, 86, 343-349.

\section{NOTE}

1. NOPE analyses presented in this paper have been programmed for use on an IBM PC. FORTRAN source code is available on diskette from the author.

(Manuscript received June 27, 1988; revision accepted for publication July $10,1989$. ) 